\title{
Exploiting the instability of smart structure for reconfiguration
}

Jiaying Zhang, Chen Zhang, Lin Hao, Rui Nie, and Jinhao Qiu

Citation: Appl. Phys. Lett. 111, 064102 (2017); doi: 10.1063/1.4986795

View online: http://dx.doi.org/10.1063/1.4986795

View Table of Contents: http://aip.scitation.org/toc/apl/111/6

Published by the American Institute of Physics

\section{Articles you may be interested in}

Direct observation of spoke evolution in magnetron sputtering

Applied Physics Letters 111, 064103 (2017); 10.1063/1.4994192

Homogenizing microwave illumination in thermoacoustic tomography by a linear-to-circular polarizer based on frequency selective surfaces

Applied Physics Letters 111, 063703 (2017); 10.1063/1.4993942

Electronically steerable ultrasound-driven long narrow air stream

Applied Physics Letters 111, 064104 (2017); 10.1063/1.4985159

Thermoelectric and thermospin properties in a quantum ring with an embedded protein-like single-helical molecule

Applied Physics Letters 111, 063701 (2017); 10.1063/1.4986380

Frequency transitions in phononic four-wave mixing

Applied Physics Letters 111, 064101 (2017); 10.1063/1.4985266

Enhanced ferro- and piezoelectric performances through interface control in $\mathrm{BiFe}_{0.99} \mathrm{~W}_{0.01} \mathrm{O}_{3} /$

$\mathrm{BiFe}_{0.95} \mathrm{Mn}_{0.05} \mathrm{O}_{3} / \mathrm{BiFe}_{0.99} \mathrm{~W}_{0.01} \mathrm{O}_{3}$ thin film

Applied Physics Letters 111, 062902 (2017); 10.1063/1.4998162

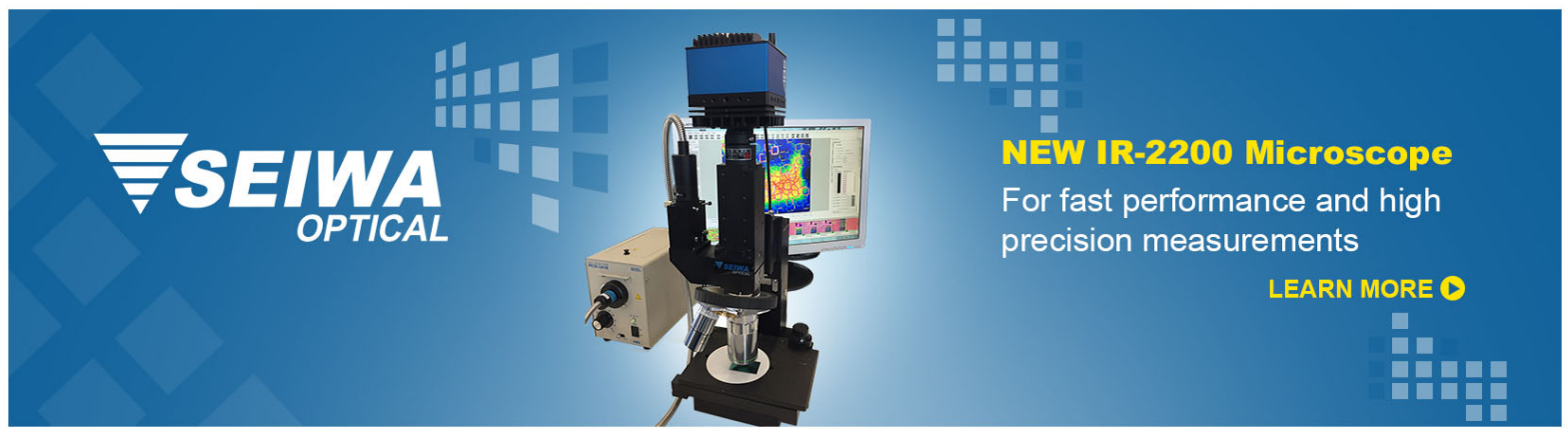




\title{
Exploiting the instability of smart structure for reconfiguration
}

\author{
Jiaying Zhang, ${ }^{1,2, a)}$ Chen Zhang, ${ }^{3}$ Lin Hao ${ }^{3}$ Rui Nie, ${ }^{3}$ and Jinhao Qiu ${ }^{3}$ \\ ${ }^{1}$ Department of Mechanical and Aerospace Engineering, University of Strathclyde, Glasgow G1 IXJ, \\ United Kingdom \\ ${ }^{2}$ School of Engineering, University of Glasgow, Glasgow G12 8QQ, United Kingdom \\ ${ }^{3}$ State Key Laboratory of Mechanics and Control of Mechanical Structures, Nanjing University \\ of Aeronautics and Astronautics, Yudao Street 29, Nanjing 210016, People's Republic of China
}

(Received 6 June 2017; accepted 27 July 2017; published online 9 August 2017)

\begin{abstract}
Aiming to verify the concept of using heteroclinic connections to reconfigure smart structures, a multistable buckled beam with integrated Shape Memory Alloy (SMA) wires is utilized as a high fidelity model. The Shape Memory Alloy (SMA) wires are resistively heated to provide the actuation force to stabilize the unstable configuration and the transition of the beam from one unstable equilibrium condition to the other. This concept provides a means of reducing the energy requirement for transitions between configurations of the structure, which is an energy-efficient reconfiguration scheme between equal-energy unstable (but actively controlled) equilibria. This letter presents a detailed design of the system, and how the active (heated) SMA wires control the structure stay in unstable configuration and drive the structure to achieve reconfiguration. Exploiting the instability of the smart structure has significant interests in many power reduction applications, including active flow control, reconfiguration of large deployable aerospace structures, and MEMS devices. Published by AIP Publishing. [http://dx.doi.org/10.1063/1.4986795]
\end{abstract}

Many physical systems in the natural world exhibit multiple stable characteristics, ${ }^{1}$ helical shapes,${ }^{2,3}$ reconfigurable property, ${ }^{4}$ deployable property, ${ }^{5}$ or foldability. ${ }^{6}$ For example, the Venus flytrap leaf can be triggered in $100 \mathrm{~ms}$ to capture insects to rapidly snap through from an open to a closed state, and the fast movement of the trap results from a snapbuckling instability which is controlled actively by the plant. ${ }^{1}$ Indeed, the multistable characteristic also plays a key role in engineering applications, such as reconfigurable structures, ${ }^{7}$ shape-changing mirrors in adaptive optical systems, ${ }^{8}$ artificial muscles, ${ }^{9}$ bioinspired robots, ${ }^{10}$ and energy harvesting. ${ }^{11}$ To implement the change between different stable states, researchers have been utilising the smart materials to change the property of the system ${ }^{10,12}$ or to drive the mechanism directly. ${ }^{13}$ Some bistable compliant mechanisms are integrated with smart materials to switch between different stable positions. ${ }^{12,14}$ A typical smart material is the shape memory alloy (SMAs), which is also made of SMA wires to embed or joint with structures for the active control. ${ }^{15-18}$ Current multistable structures have been largely utilized to satisfy manifold engineering applications, from largescale morphing aerofoils ${ }^{19}$ to MEMs-scale switches. ${ }^{20}$ Moreover, a recently developed concept utilizes the multistable property to design a monolithic mechanical metamaterials. ${ }^{21}$ Meanwhile, actively controlled reconfigurable structures and mechanisms have been exploited in the design of different requirements, including the design of a special class of acoustic waveguides, ${ }^{22}$ self-folding machines, ${ }^{23}$ and shapemorphing microarchitectured materials. ${ }^{24}$ Furthermore, an advanced application has been investigated to synthesise the multistability by connecting bistable units together. ${ }^{25}$ Nonlinear behaviour in mechanical multistable structures related with bifurcation phenomena ${ }^{26}$ and some key parameters could be

\footnotetext{
${ }^{\text {a)} E-m a i l: ~ j i a y i n g . z h a n g @ s t r a t h . a c . u k ~}$
}

proposed to switch between different functional configurations upon actuation. ${ }^{27}$

In general, nonlinear dynamical systems typically possess a number of equilibria which are stable and unstable. These equilibria could be connected through the paths in the phase space of the system, and the special one is called heteroclinic connections which connect both stable and unstable manifolds of equilibria. The traditional concept of morphing structures is between stable configurations, which requires the input of, and then the dissipation of energy to cross the potential barrier separating the stable equilibria. However, to overcome the addition of or dissipation of energy to cross a potential barrier, reconfiguration between equal-energy unstable states can be achieved for conserving energy, which is verified by numerical methods in a simple smart structure model. ${ }^{27,28}$ Clearly, energy is required to stabilize the unstable configurations, but if the energy required to actively control the instability is sufficiently small or devices need to be frequently switched between different states, this concept is likely to be of benefit for reconfiguring. The accumulated work done of frequently actuated devices in reconfiguring between stable states will be significant, while the duration of the system spends in the active control for stabilizing the unstable states will be less. The active control of unstable smart structures has been investigated by using an agentbased approach for reducing the energy requirements. ${ }^{29}$ Simulation results show that reconfiguration between such unstable equilibria can be energetically more efficient compared to transitions between stable configurations. ${ }^{27-29}$ The previous numerical model is considered as an elastic beam clamped at both ends and represented as masses connected to linear springs. Then, the result shows that the structure can be actively controlled in an unstable state, and the unstable configuration of the structure can be connected through heteroclinic connections in the phase space. ${ }^{27,30}$ 
In order to establish the use of instability for developing future practical devices, this Letter pursues a laboratory experiment that builds on prior simulation-based work. It is our intention in this letter to present this concept with a numerical simulation and experimental demonstration which are based on the previous simulation results. ${ }^{30-32}$ Heteroclinic connections will be investigated here as a rigorous means of enabling transitions between unstable configurations of a reconfigurable smart structure. The both ends of SMA wires are fixed with a built-in pin-joints on the beam, which can be actively heated based on the means of Joule effects under an electrical current. Thus, the buckled beam can be stabilized in the two unstable equilibria [Fig. 1(a)] and reconfigured by transiting different geometry configurations between these two unstable equilibria [Fig. 1(b)]. It is obvious that the moving beam has a specific geometry configuration at any moment during the transition process. Meanwhile, different geometry configurations could change the midplane stretching of the beam, which will influence the support force at both ends of the beam. Therefore, the load curve could be used to verify experimental results that are in a good agreement with the numerical prediction. While future work will allow for comparison of energy consumption between reconfiguration between stable configurations and reconfiguration between unstable configurations, this work serves as an initial demonstration that it is a possible way to reconfigure a high fidelity model between unstable states in an experimental setup.

The first critical load according to the first beam buckling mode is ${ }^{11}$

$$
P_{c r}^{(1)}=\frac{4 \pi^{2} E I}{l_{0}^{2}},
$$

where $E$ is Young's modulus, $I$ is the moment of inertia of the cross section, and $l_{0}$ is the initial length of the beam. The second buckling critical load is greater than the first cortical load according to the second beam buckling mode is

$$
P_{c r}^{(2)}=\frac{8.18 \pi^{2} E I}{l_{0}^{2}} .
$$

The stability of these buckled configurations can be investigated by an analytical method, ${ }^{33}$ and the result shows that the first buckled configuration is a stable equilibrium position other than the second buckled configuration. The buckled beam has the ability to snap from one stable state to the other when excited with sufficient input actuation. Remarkably, for
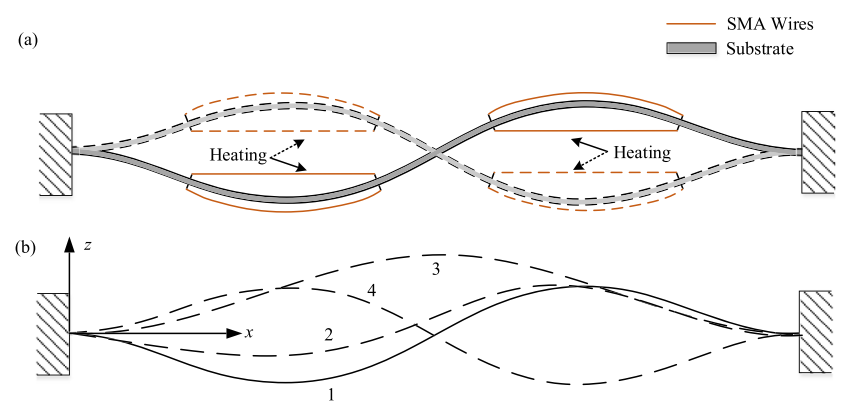

FIG. 1. The buckled beam model. (a) Schematic of an unstable buckedbeam and (b) different stages (1-2-3-4) during the reconfiguration. frequently switched devices, the accumulated work done will be significant in reconfiguring between stable equilibria, while the system depends on the active control at unstable equilibria will be small. An numerical simulation result is shown in Fig. 2 based on the governing equation [Eq. (3)] with nondimensional quantities ${ }^{33}$

$$
\ddot{w}+w^{\prime \prime \prime \prime}+P w^{\prime \prime}+c \dot{w}-\frac{1}{2} w^{\prime \prime} \int_{0}^{1} w^{\prime 2} d x=0,
$$

where the overdot indicates the derivative with respect to time $\tau$, the prime indicates the derivative with respect to $x$, and $P$ and $c$ are nondimensional quantities of axial force and the viscous damping coefficient, respectively. According to the analytical results of buckling problem, the corresponding first buckling mode shape is given by

$$
w_{c r}^{(1)}=c_{1}(1-\cos (2 n \pi x)),
$$

and the second buckling mode is

$$
w_{c r}^{(2)}=c_{2}(1-2 x-\cos (\lambda x)+2 / \lambda \sin (\lambda x)),
$$

where $c_{1}$ and $c_{2}$ is a constant.

Then, in order to verify the heteroclinic connection between the unstable buckling configurations, one of the second buckling mode from Eq. (5) is used as the initial condition with small perturbation to solve the governing equation Eq. (3) by simply omitting nonlinear terms $-\frac{1}{2} w^{\prime \prime} \int_{0}^{1} w^{\prime 2} d x$ and damping term $c \dot{w}$. The open-source package Chebfun ${ }^{34}$ is used to obtain the numerical solution, which is shown in Fig. 2.

Figure 2 shows the deflection of the buckled beam during reconfiguration between two unstable buckling modes $\left(E_{i}^{u}\right.$, $E_{e}^{u}$ ), and it is distinct that this transition goes through a stable mode $\left(E^{s}\right)$. Moreover, in order to explain that the heteroclinic connection between unstable equilibria is existed in such a partial differential system, the system is projected to a phase space. In this way, we can simply observe the dynamics of states and the main trends of the transition between states. This phase space of this dynamic system depicts all the possible shapes of the beam and consists of two values ( $w_{1}$ and $w_{2}$ ), which means the deflection of stationary points. Then, the system's evolving state over time traces a phase space trajectory, which connects the initial states and final states. In this problem, the initial and final unstable configuration $\left(E_{i}^{u}\right.$

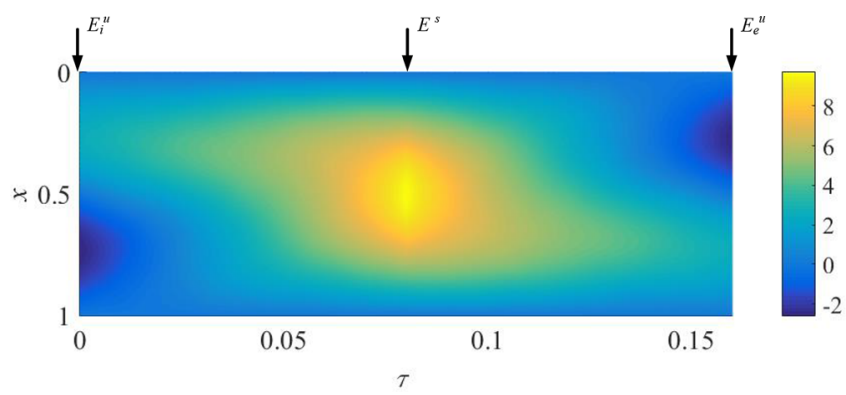

FIG. 2. Contour plot of the deflection history during reconfiguration. $\tau$ is the nondimensional time. $E_{i}^{u}, E^{s}$, and $E_{e}^{u}$ are the initial unstable configuration, the in-between stable configuration, and the terminal unstable configuration, respectively. 


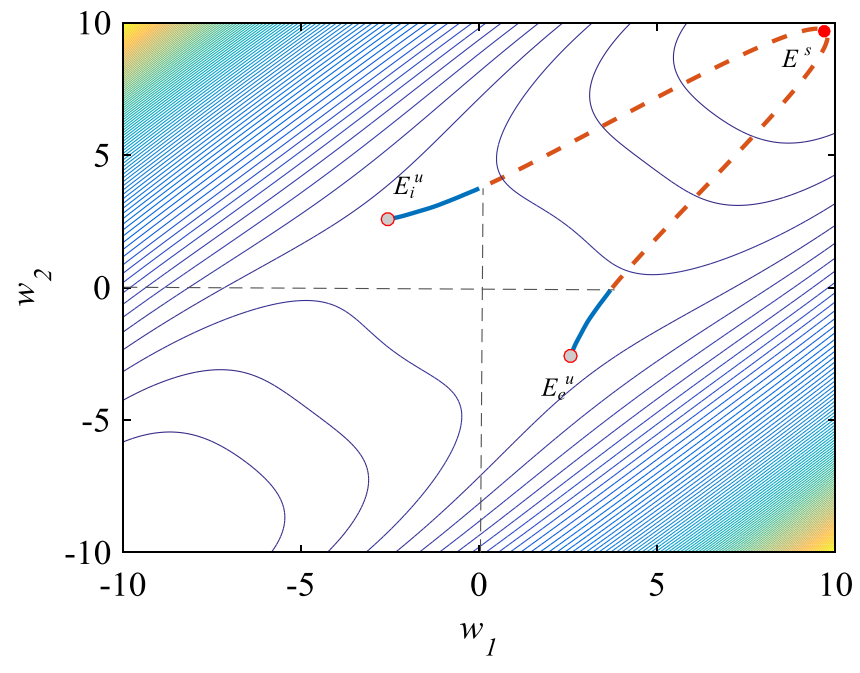

FIG. 3. The schematic diagram of the heteroclinic connection between two distant equilibria $E_{i}^{u}$ and $E_{i}^{u}$ in assumed phase space. $w_{1}$ and $w_{2}$ are the two extrema of deflection during reconfiguration.

and $E_{e}^{u}$ ) could be displayed as a point in the phase space with $w_{1}=-w_{2}$, and the stable configuration $\left(E^{s}\right)$ is displayed as a point with $w_{1}=w_{2}$. The two stationary points first transfer to the midpoint and then part to opposite directions, which can be regarded as a trajectory (heteroclinic connection) depart from one unstable equilibrium $E_{i}^{u}$ to another unstable equilibrium $E_{e}^{u}$ by crossing a stable equilibrium $E^{s}$, as shown in Fig. 3. It is obvious that the heteroclinic connection is a highly efficient transition with low energy dissipation when compared to other transitions.

A beam prototype is fabricated by acrylic with their geometry property listed in Table I. The fabricated device is composed of an acrylic beam, partly jointed with SMA wires and fixed at both ends. Thermos-mechanical properties of the SMA are reported in Table II.

The actuating force is based on the thermo-mechanical properties of SMAs, which is due to a crystallographic phase transformation (austenite to martensite, or vice versa). From the view of the macroscopic scale, after distorting the SMAs, the original shape can be recovered simply by heating the wire above the temperature $A_{f}$. The heat transferred to the wire is the power driving the molecular rearrangement of the alloy, which is configured in the original shape of the wire. Young' modulus of the material can be obtained by a basic constitutive relation

$$
E=\sigma / \varepsilon=(\Delta F / w \cdot t) /\left(\Delta s / l_{0}\right)
$$

where $\sigma$ is stress, $\varepsilon$ is the strain, $w$ is the width, $t$ is the thickness, and $l_{0}$ is the initial length of the beam. $\Delta F$ and $\Delta s$ are load and displacement measured by the testing machine.

TABLE I. Geometry property of the proposed beam.

\begin{tabular}{lc}
\hline \hline Description & Value \\
\hline Substrate beam length $(\mathrm{mm})$ & 400 \\
Substrate beam width $(\mathrm{mm})$ & 25 \\
Substrate beam thickness, $(\mathrm{mm})$ & 2 \\
\hline \hline
\end{tabular}

TABLE II. Selected SMA materials properties.

\begin{tabular}{lc}
\hline \hline Description & Value \\
\hline Diameter, $d(\mathrm{~mm})$ & 0.5 \\
Martensite Young modulus, $E_{M}(\mathrm{GPa})$ & 22 \\
Austenite Young modulus, $E_{A}(\mathrm{GPa})$ & 50.3 \\
Martensite start temperature, $M_{S}\left({ }^{\circ} \mathrm{C}\right)$ & 18.4 \\
Martensite finish temperature, $M_{f}\left({ }^{\circ} \mathrm{C}\right)$ & 9 \\
Austenite start temperature, $A_{s}\left({ }^{\circ} \mathrm{C}\right)$ & 32 \\
Austenite finish temperature, $A_{f}\left({ }^{\circ} \mathrm{C}\right)$ & 42 \\
Critical stress de-twinned martensite start $(\mathrm{MPa})$ & 100 \\
Critical stress de-twinned martensite finish $(\mathrm{MPa})$ & 170 \\
Thermal expansion coefficient $\Theta\left(\mathrm{MPa} /{ }^{\circ} \mathrm{C}\right)$ & 0.55 \\
Variation of austenite critical temperature $C_{A}$ with stress $\left(\mathrm{MPa} /{ }^{\circ} \mathrm{C}\right)$ & 13.8 \\
Variation of martensite critical temperature $C_{M}$ with stress $\left(\mathrm{MPa} /{ }^{\circ} \mathrm{C}\right)$ & 8 \\
Limit strain $\varepsilon_{L}(\%)$ & 7.2 \\
\hline \hline
\end{tabular}

A series of experiment were carried out to investigate the effectiveness of the proposed beam stabilized in an unstable configuration and reconfiguration between different unstable states, which is described as the static and dynamic behaviour of the combined system comprising of a beam and SMA wires. The numerical analysis of the analogous nonlinear system is shown in Ref. 31; a heteroclinic connection is verified between two unstable states, and the geometry of the transition process is obtained through simulation, as shown in Figs. 1(b) and 2. In the present study, the aforementioned slender beam is clamped at both ends on the testing machine, as shown in Fig. 4(a). In general, unstable buckled configurations will never occur because of its instability, but it is easy to actively control this unstable buckled configuration through heating the selected SMA wires. Therefore, the initial unstable configuration of the beam could appear when the load is acting through the testing machine, as shown in Fig. 4(b). The final unstable configuration is shown in Fig. 4(c), which is the symmetric geometry with initial configuration under active control.

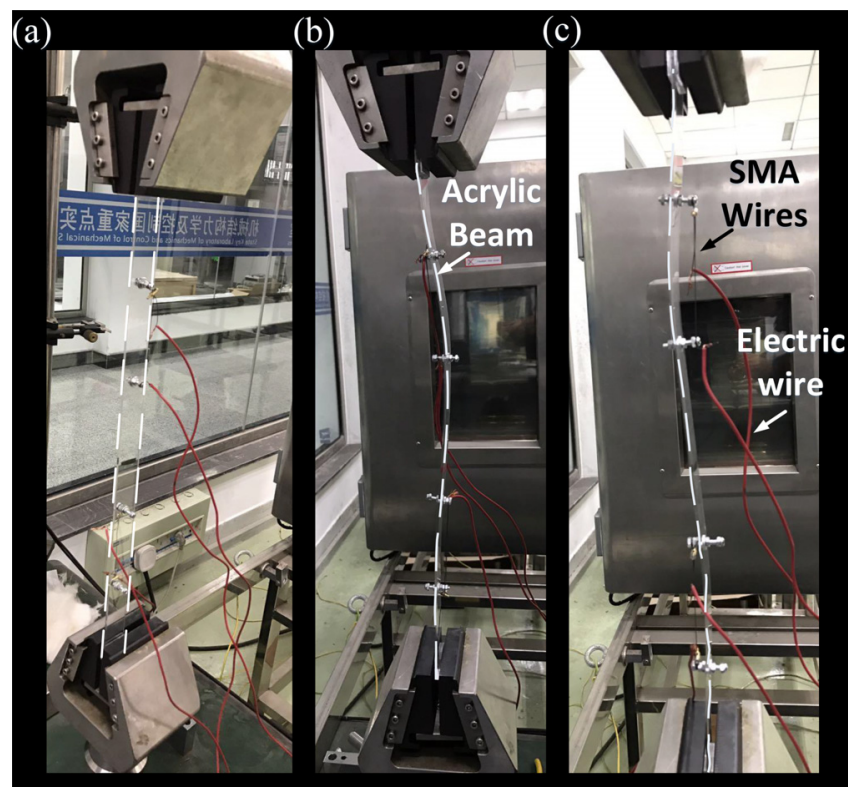

FIG. 4. Photograph of the buckled beam: (a) acrylic beam without load, (b) initial unstable configuration, and (c) final unstable configuration. 
In order to illustrate the experimental result could fulfil a good agreement with the numerical prediction, the load at both ends of the beam is calculated by the numerical method. According to Ref. 35, the beam's midplane stretching is shown as

$$
\Delta=u(L)-u(0)+\frac{1}{2} \int_{0}^{L}\left(\frac{\partial w}{\partial x}\right)^{2} d x
$$

where $u(L)$ and $u(0)$ are the axial displacements at the ends of the beam. In this model, the ends of the beam are fixed at both ends. Therefore, the induced axial force can be expressed as follows:

$$
N=\frac{E A}{L} \Delta=\frac{E A}{2 L} \int_{0}^{L}\left(\frac{\partial w}{\partial x}\right)^{2} d x
$$

where $E A / L$ is the axial stiffness of the beam. The support load in the both ends of the beam then can be calculated when the beam is reconfiguring between different configurations. Figure 5 illustrates the load curves during the reconfiguration obtained by numerical methods and experimental methods; three stages are arranged to explain the reconfiguration scheme. The first stage is the loading procedure that the testing machine compress the structure to produce the second buckled configuration, and the value of the beam's second critical load is almost $14.5 \mathrm{~N}$ here $(8.97 \%$ error with the theoretical value). This unstable configuration is then stabilized through the ohmic heating of selected SMA wires. The second stage is reconfiguration, which switches the active control of target SMA wires and the testing machines suspended to ensure no extra load and displacement. The third stage is similar to the first one, which is the load procedure.

A sudden drop of the load is observed in the second stage and could not return to the value of load before

(a)
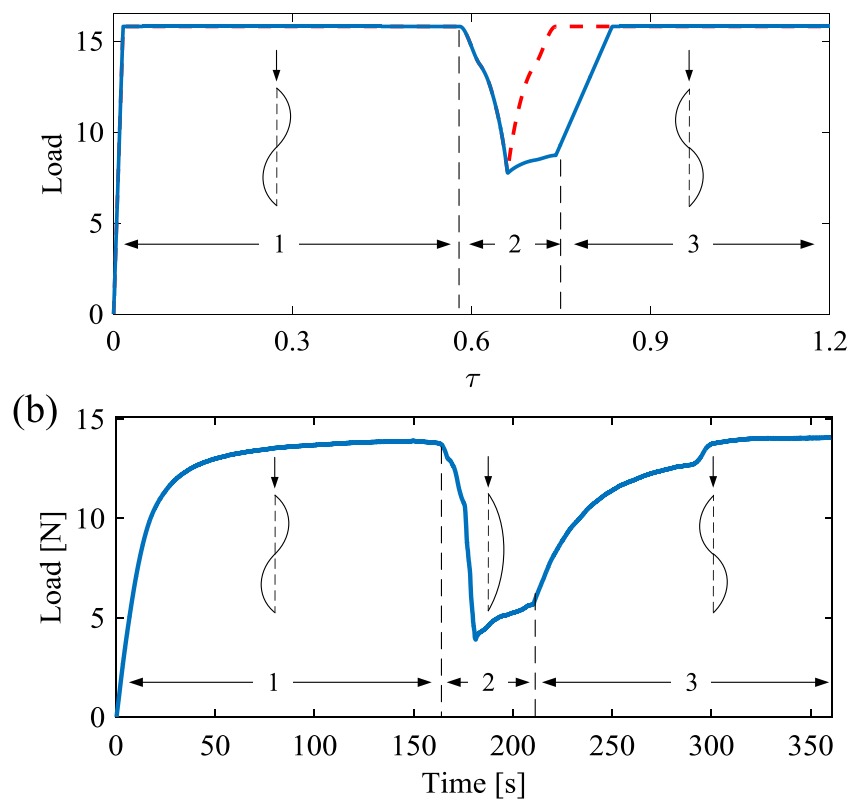

FIG. 5. Load graph during reconfiguration with three stages. (a) Numerical result: solid line-with residual tensile force and dashed line-without residual tensile force. (b) Experimental result. reconfiguration. The reason for the sudden drop is that the structure is moving through the stable configuration $E^{s}$ [as shown in Fig. 1(b)], which is the first buckled configuration with the lower critical load. Figure 5(a) shows that two load curves are based on different situations; the solid line and dashed line represent with the residual tensile force and without the residual tensile force, respectively. However, the residual tensile force cannot be ignored in the practical situation, which will clearly influence return tendency of the load curve as shown in Fig. 5(b). Therefore, the third stage is arranged to guarantee the reconfiguration between unstable configurations that are fully done. The third stage indicates that the structure returns to another unstable configuration, which has the same critical load with the first stage. The experiment results shown in this work are in good agreement with the numerical prediction, and they demonstrate that the heteroclinic connection between unstable states could be utilized for reconfiguring structures.

A concept for the reconfiguration of smart structures has been investigated through using the heteroclinic connections, which provides a pivotal way in reducing the energy requirement for a transition between configurations of the structure. For more practical designs, it is desirable to design structures possessing multiple equilibria offer interesting dynamical behaviour. The proposed structure was fabricated and tested to demonstrate its effectiveness for exploiting the instability of structure for reconfiguration. The smart beam fabricated in this work offers the possibility of active control as a mechanical system through the use of testing machine and sensors and shape memory alloys, which are used as sensors and actuators, respectively. The internal force of the SMAs wires helps both to stabilize the unstable configuration of the beam and trigger the reconfiguration between configurations of the beam. Finally, the results presented provide strong confidence that instability of structure can be controlled, and the structure can be reconfigured between different unstable states, with multi-physics effects, e.g., thermos-mechanical materials. It is our intention in this letter to verify this concept and provide a detailed analysis using a high fidelity model of a real structure. Additionally, it is possible to apply the instability to achieve the reconfiguration of structures or mechanisms include use in MEMS-type devices which require frequent switching to reduce mean power consumption and waste heat dissipation. It is likely to be of benefit for power and energy constrained applications, such as Aerospace and Marine sectors.

This work was supported by John Moyes Lessells Travel Scholarships of The Royal Society of Edinburgh and National Natural Science Foundation of China No. 11372133. The authors thank Professor C. R. McInnes for earlier discussions.

${ }^{1}$ Y. Forterre, J. M. Skotheim, J. Dumais, and L. Mahadevan, "How the Venus flytrap snaps," Nature 433, 421-425 (2005).

${ }^{2}$ H. Liang and L. Mahadevan, "The shape of a long leaf," Proc. Natl. Acad. Sci. U.S.A. 106, 22049-22054 (2009).

${ }^{3}$ Y. Forterre and J. Dumais, "Generating helices in nature," Science 333, 1715-1716 (2011).

${ }^{4}$ J. J. Allen, G. R. R. Bell, A. M. Kuzirian, and R. T. Hanlon, "Cuttlefish skin papilla morphology suggests a muscular hydrostatic function for rapid changeability," J. Morphol. 274, 645-656 (2013). 
${ }^{5}$ F. Haas, S. Gorb, and R. Wootton, "Elastic joints in dermapteran hind wings: Materials and wing folding," Arthropod Struct. Dev. 29, 137-146 (2000).

${ }^{6}$ D. S. A. De Focatiis and S. D. Guest, "Deployable membranes designed from folding tree leaves," Philos. Trans. R. Soc., A 360, 227-238 (2002).

${ }^{7}$ M. P. Brenner, J. H. Lang, J. Li, J. Qiu, and A. H. Slocum, "Optimal design of a bistable switch,” Proc. Natl. Acad. Sci. U.S.A. 100, 9663-9667 (2003).

${ }^{8}$ L. Datashvili, H. Baier, B. Wei, S. Endler, and L. Schreider, "Design of a morphing skin using flexible fiber composites for space-reconfigurable reflectors," in 54th AIAA/ASME/ASCE/AHS/ASC Structures, Structural Dynamics and Materials Conference (2013), pp. 1-11.

${ }^{9}$ J. Rossiter, B. Stoimenov, and T. Mukai, "A bistable artificial muscle actuator," in IEEE International Symposium on MicroNanoMechanical and Human Science (IEEE, 2006), pp. 1-6.

${ }^{10} \mathrm{M}$. Shahinpoor, "Biomimetic robotic Venus flytrap (Dionaea muscipula Ellis) made with ionic polymer metal composites," Bioinspiration Biomimetics 6, 46004 (2011).

${ }^{11}$ Y. Zhu and J. W. Zu, "Enhanced buckled-beam piezoelectric energy harvesting using midpoint magnetic force,” Appl. Phys. Lett. 103, 041905 (2013).

${ }^{12}$ Y. Du, B. Liu, M. Xu, E. Dong, S. Zhang, and J. Yang, "Dynamic characteristics of planar bending actuator embedded with shape memory alloy," Mechatronics 25, 18-26 (2015).

${ }^{13}$ A. Erturk, Macro-Fiber Composite Actuated Piezoelectric Robotic Fish (Springer Berlin Heidelberg, 2015), pp. 255-283.

${ }^{14}$ S.-M. Yang, J.-H. Roh, J.-H. Han, and I. Lee, "Experimental studies on active shape control of composite structures using SMA Actuators," J. Intell. Mater. Syst. Struct. 17, 767-777 (2006).

${ }^{15}$ E.-J. Yoo, J.-H. Roh, and J.-H. Han, "Wrinkling control of inflatable booms using shape memory alloy wires," Smart Mater. Struct. 16, 340-348 (2007).

${ }^{16} \mathrm{H}$. Ishii and K. L. Ting, "SMA actuated compliant bistable mechanisms," Mechatronics 14, 421-437 (2004).

${ }^{17}$ S. Barbarino, F. S. Gandhi, and R. Visdeloup, "A bi-stable von-mises truss for morphing applications actuated using shape memory alloys," in Volume 1: Development and Characterization of Multifunctional Materials; Modeling, Simulation and Control of Adaptive Systems; Integrated System Design and Implementation (ASME, 2013), p. V001T01A004.

${ }^{18}$ A. Crivaro, R. Sheridan, M. Frecker, T. W. Simpson, and P. Von Lockette, "Bistable compliant mechanism using magneto active elastomer actuation,” J. Intell. Mater. Syst. Struct. 27(15), 2049-2061 (2015).
${ }^{19}$ M. E. Pontecorvo, S. Barbarino, G. J. Murray, and F. S. Gandhi, "Bistable arches for morphing applications," J. Intell. Mater. Syst. Struct. 24, 274-286 (2013).

${ }^{20}$ M. D. Williams, K. F. Van, and M. Sheplak, "Modeling of Initially curved beam structures for design of multistable MEMS," J. Appl. Mech. 79, 11006 (2012).

${ }^{21}$ A. Rafsanjani, A. Akbarzadeh, and D. Pasini, "Snapping mechanical metamaterials under tension," Adv. Mater. 27, 5931-5935 (2015).

${ }^{22}$ S. Babaee, J. T. B. Overvelde, E. R. Chen, V. Tournat, and K. Bertoldi, "Reconfigurable origami-inspired acoustic waveguides," Sci. Adv. 2, e1601019 (2016).

${ }^{23}$ S. Felton, M. Tolley, E. Demaine, D. Rus, and R. Wood, "A method for building self-folding machines," Science 345, 644-646 (2014).

${ }^{24}$ L. A. Shaw and J. B. Hopkins, "An actively controlled shape-morphing compliant microarchitectured material," J. Mech. Rob. 8, 21019 (2015).

${ }^{25}$ Y. S. Oh and S. Kota, "Synthesis of multistable equilibrium compliant mechanisms using combinations of bistable mechanisms," J. Mech. Des. 131, 21002 (2009).

${ }^{26}$ Z. Chen, Q. Guo, C. Majidi, W. Chen, D. J. Srolovitz, and M. P. Haataja, "Nonlinear geometric effects in mechanical bistable morphing structures," Phys. Rev. Lett. 109, 114302 (2012).

${ }^{27}$ J. Zhang and C. R. McInnes, "Using instability to reconfigure smart structures in a spring-mass model," Mech. Syst. Signal Process. 91, 81-92 (2017).

${ }^{28}$ J. Zhang and C. R. McInnes, "Reconfiguring smart structures using approximate heteroclinic connections," Smart Mater. Struct. 24, 105034 (2015).

${ }^{29}$ O. Guenther, T. Hogg, and B. A. Huberman, "Controls for unstable structures," Proc. SPIE 3039, 754-763 (1997).

${ }^{30}$ C. R. McInnes and T. J. Waters, "Reconfiguring smart structures using phase space connections," Smart Mater. Struct. 17, 25030 (2008).

${ }^{31}$ J. Zhang and C. R. McInnes, "Reconfiguration of a four-bar mechanism using phase space connections," Mech. Syst. Signal Process. 81, 43-59 (2016).

${ }^{32}$ J. Zhang, C. R. McInnes, and M. Xu, "Reconfiguration of a smart surface using heteroclinic connections," Proc. R. Soc. A 473, 20160614 (2017).

${ }^{33}$ A. H. Nayfeh and S. A. Emam, "Exact solution and stability of postbuckling configurations of beams," Nonlinear Dyn. 54, 395-408 (2008).

${ }^{34}$ Z. Battles and L. N. Trefethen, "An extension of MATLAB to continuous functions and operators," SIAM J. Sci. Comput. 25, 1743-1770 (2006).

${ }^{35}$ A. H. Nayfeh and D. T. Mook, Nonlinear Oscillations (Wiley-VCH Verlag GmbH, Weinheim, Germany, 1995). 